\section{A FORTRAN procedure for plotting two-dimensional barycentric simplicia}

\author{
JAMES E. HAYS \\ Puget Sound Blood Center, Terry at Madison \\ Seattle, Washington 98104
}

The results of much research, particularly in the behavioral sciences, is presented in the form of contingency tables, which, until the introduction of Snee's (1974) procedure for graphic display, had not lent themselves easily to graphical summary. Such summary is particularly appropriate when a significant difference between distributions is found. When a significant effect is observed in an $\mathrm{R}$ by $\mathrm{C}$ table, it then becomes of interest to determine which rows are producing the nonhomogeneity. Since each of the rows is a sample from a multinomial distribution, each observed frequency $n_{i j}$ can be expressed as a proportion $\mathrm{p}_{\mathrm{ij}}$ of the total row frequency $\mathrm{N}_{\mathrm{i}}$. Each row can then be plotted as a point in a $\mathrm{C}-1$ dimensional simplex. Points which cluster together indicate that the associated rows are homogeneous. The $100(1-\alpha)$ confidence interval is approximated by the set of all points $\left(a_{i 1}, a_{i 2}, \cdots, a_{i C}\right)$ for which

$$
\frac{p_{i 1}^{2}}{a_{i 1}}+\frac{p_{i 2}^{2}}{a_{i 2}}+\cdots+\frac{p_{i C}^{2}}{a_{i C}} \leqslant\left(\frac{w}{N_{i}}+1\right)
$$

where $p_{i j}$ is the proportion of the sample $N_{i}$ in the $j^{\text {th }}$ category, $w$ is the $100(1-\alpha)$ percentile of the chi-square distribution with $\mathrm{C}-1$ degrees of freedom and $\mathbf{N}_{i}$ is the total sample size.

The procedure that Snee developed can be easily applied to one-dimensional simplicia and results in a simple bar graph. Confidence intervals are likewise easily calculated and displayed.

For $\mathrm{R}$ by 3 tables, display of the corresponding twodimensional simplex becomes somewhat more difficult. Although triangular coordinate paper is available commercially to facilitate the plotting of two-dimensional barycentric simplicia, the subsequent plotting of confidence intervals is extremely laborious, involving the evaluation of all points in the space and plotting those for which the above equation is satisfied.

A FORTRAN computer procedure has been written to facilitate the display of such two-dimensional simplicia. The program plots specific points and confidence intervals in the rectangular coordinate system available on most line printers. The output may be scaled to be compatible with the printing space on any commerically available line printer.

The rectangular coordinates corresponding to the point $\left(p_{1}, p_{2}\right)$ in barycentric space are:

$$
y=V-p_{1}(V-1),
$$

where $V=$ the vertical size of the plotting space in characters and

$$
x=(H-1)\left(p_{2}+1 / 2 p_{1}\right)+2,
$$

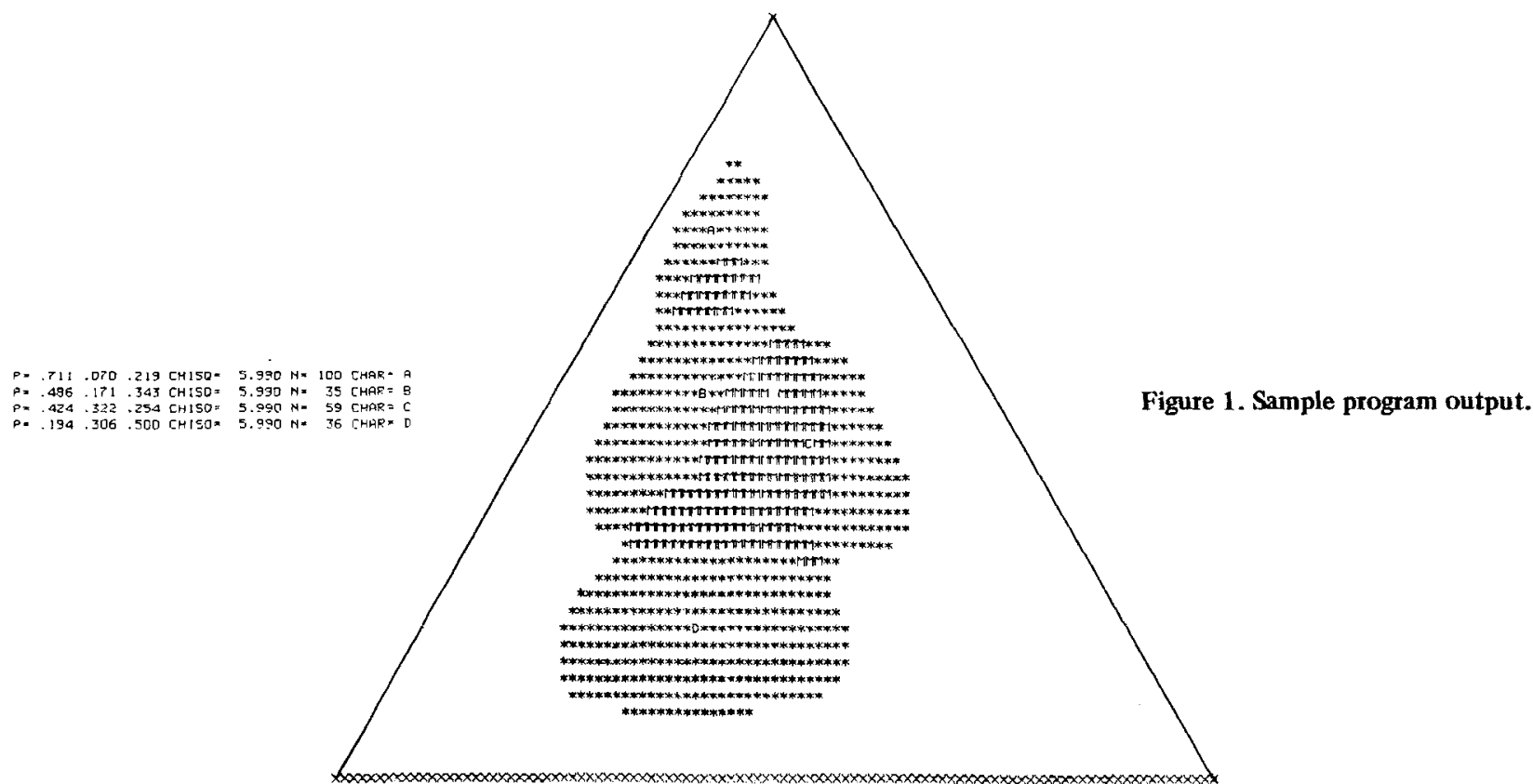


where $\mathrm{H}=$ the horizontal size of the plotting space in characters.

Input. The procedure consists of a FORTRAN main program and three subroutines. Input is read from cards and consists of the following parameters: (1) The chart width and height/width ratio that are appropriate for the line printer being used. Height/width ratios are specified in terms of the number of characters per inch of height to the number of characters per inch of width. Chart width is in terms of the total width in characters. (2) Input of specific points to be plotted is read from cards and consists of the $p_{1}$ and $p_{2}$ values for a point, the $w$ value for the confidence interval desired, the number of observations for that point, and a plotting character. If an illegal point is entered (i.e., $1-p_{1}$ $-\mathrm{p}_{2}<0$ ), that point is rejected and another card is read. Any number of points may be plotted on the same set of coordinates. The printout of the plotting space occurs when a blank card is read.
Output. The printed output consists of a twodimensional barycentric plot and a listing of all points plotted. The actual point is plotted with the character chosen by the user; points within the confidence interval are plotted with asterisks. Multiple points are plotted with an $M$.

Computer and Language. The program was written in FORTRAN and has been tested on a Raytheon 704. Figure 1 is an example of the output produced by the program for the data given by Snee, Table 2 .

Availability. A listing of the program, test data, sample output, and instructions may be obtained free of charge from James E. Hays, Puget Sound Blood Center, Seattle, Washington.

\section{REFERENCE}

SNEE, R. D. Graphical display of two-way contingency tables. The American Statistician. 1974, 28, 9-12. 